\title{
Is Viral Co-infection A Risk Factor For Severe Lower Respiratory Tract Infection? A Retrospective Observational Study
}

\author{
Aykut Eşki ${ }^{1}$, Gökçen Kartal Öztürk ${ }^{2}$, Candan Çiçek ${ }^{3}$, Figen Gülen ${ }^{4}$, and Esen Demir ${ }^{4}$ \\ ${ }^{1}$ Diyarbakır Gazi Yaşargil Eğitim ve Araştırma Hastanesi \\ ${ }^{2}$ Ege University Faculty of Medicine \\ ${ }^{3}$ Ege Universitesi Tip Fakultesi \\ ${ }^{4}$ Ege University Faculty of Medicine, Department of Pediatrics, Division of Pulmonology
}

January 21, 2021

\begin{abstract}
Objective: To determine whether viral coinfection is a risk for severe lower respiratory tract infection (LRTI). Working Hypothesis: Children with viral coinfection had a higher risk for admission to the intensive care unit (ICU) than those with a single virus infection. Study Design: Retrospective, observational study for ten years. Patient-Subject Selection: Children between 1-60 months of age hospitalized with LRTI. Methodology: We defined severe LRTI as admission to the ICU for high-flow nasal cannula oxygen/bilevel positive airway pressure/invasive ventilation and assessed demographic and laboratory data with potential risk factors from the patients' medical records. Results: Of 2115 children hospitalized with LRTI, 562 had severe, and 1553 had mild disease. Viral coinfection was present in $28.3 \%$ of all patients, and those with viral coinfection were at a higher risk of severe LRTI than those with a single virus infection (43.8\% vs. 22.7\%; aOR, 3.44; 95\% CI, 2.74-4.53). Respiratory syncytial virus (RSV) and rhinovirus (except for between 25-60 months) coinfections were associated with severe LRTI in all ages, whereas parainfluenza virus-3 (PIV3; 7-24 months) and bocavirus (7-12 months) coinfections led to severe LRTI in early childhood. Moreover, influenza-A coinfection caused severe LRTI in children between 7-12 and 25-60 months. Other risk factors included young age, prematurity, history of atopy, exposure to tobacco smoke, underlying condition, neutrophilia, lymphopenia, and high CRP value. Conclusion: Children with viral coinfection, particularly with rhinovirus, RSV, influenza-A, PIV3, and bocavirus, may be followed closely regarding the clinical changes.
\end{abstract}

\section{1-INTRODUCTION}

Viral lower respiratory tract infection (LRTI) presents with an acute, self-limiting, and uncomplicated infection in healthy children, with a significant cause of public health problems and health care costs in those with any known risk factor ${ }^{1}$. In the United States (US), pneumonia and bronchiolitis, generally associated with viral agents, are the most common causes of LRTI-associated hospitalizations in children under five years $^{2}$. Moreover, between 2002-2009, the estimated hospital charges for children with bronchiolitis younger than two years of age increased from 1.3 to 1.7 billion $^{3}$.

With the advances in molecular technology, multiple viruses have been determined at a rate of $30-70 \%$ in children hospitalized with $\mathrm{LRTI}^{4,5}$. However, the clinical implications of viral coinfection remain unclear and controversial reports have been published. While Richard et al. ${ }^{6}$ found that infants with the dual respiratory virus were 2.7 times higher at risk of admission to the intensive care unit (ICU) than those with single-virus infection, Brand et al. ${ }^{7}$ showed no increased risk of severe bronchiolitis in infants with multiple viruses. Despite the small sample size, our previously published study suggested that the presence of influenza A and human bocavirus (HBoV) coinfection were independently a risk for invasive mechanical ventilation (IMV) support $^{8}$. Some meta-analyses showed a comparable effect on hospital admission, the length of hospital stay, 
and oxygen requirements or death between children with a single virus and viral coinfection ${ }^{5,9}$. In contrast, a systemic review determined that further research has needed to clarify the impact of viral coinfection on the severity of LRTI because of conflicting results in studies ${ }^{10}$.

Considering these controversial reports, we conducted an observational study to investigate the effect of virus coinfection on LRTI in hospitalized children under five years of age. These findings may identify the degree to which clinicians should consider viral coinfection when assessing children's risk of developing severe illness.

\section{2-METHODS}

\section{1-Study design, sites, and subjects}

We performed a single-center retrospective study of children under five years of age hospitalized with a clinical diagnosis of LRTI, presenting to the emergency department, outpatient clinics (pediatric pulmonology, pediatrics, pediatric infectious disease), or ICU of Ege University Medical Faculty Children Hospital from January 2010 through January 2020. The hospital institutional review board approved this study.

The study institution's standard practice is to screen all admitted patients with respiratory symptoms for viral respiratory pathogens. Because the International Classification of Diseases discharge codes might be inaccurate coding or misdiagnosis on the part of the treating clinician, cases were identified by evaluating the virology laboratory database. Subsequently, we cross-checked the virology laboratory results with electronic medical records and nurse observation charts. According to the revised World Health Organization guidelines ${ }^{11}$, patients between 1-60 months of age who clinically fulfilled the criteria of LRTI (pneumonia or bronchiolitis) were included in the study. Exclusion criteria included hospitalization within the preceding 30 days, other diagnoses than LRTI caused by respiratory viruses, hospital-acquired viral LRTI, primary (defined as bacterial growth detected in cultures from sterile cavities such as blood, cerebrospinal fluid, urine, and pleural fluid) or secondary (defined as bacterial growth in cultures taken from sterile cavities 48 hours after hospitalization) ${ }^{12}$ bacterial infections, newborns (under one month of age), and incomplete medical records.

\section{2-Data collection for risk factors, clinical course, and outcomes}

We reviewed risk factors, clinical course, and outcomes of all patients eligible for the study. The potential risk factors for severe LRTI regarding the literature ${ }^{7,13-17}$ were abstracted from the electronic medical record. The age, gender, prematurity ( $<37$ weeks), history of atopy (clinician-confirmed atopy), exposure to tobacco smoke (smokers in the household), malnutrition status (assessed with weight-for-height standard deviation score [SDS] $)^{18}$, the number and type of virus, underlying condition (immunosuppression [IS], neuromuscular disease [NMD], chronic lung disease [CLD], hemodynamically significant congenital heart disease [CHD], and previous history of LRTI), neutrophil and lymphocyte status for age ${ }^{19}$, c-reactive protein $(\mathrm{CRP})$ value, oxygen requirement (defined as peripheral oxyhemoglobin saturation $<92 \%$ ), and admission to the ICU (defined as those admitted if they required high-flow nasal cannula oxygen [HFNCO]/bilevel positive airway pressure $[\mathrm{BiPAP}] / \mathrm{IMV})$ or short term unit were collected. Children with multiple underlying conditions were included in the group considered having a higher risk for underlying disease. Patients with IS/NMD considered the highest-risk, followed by CLD, CHD, and then the previous history of LRTI.

\section{2-Viral diagnostic testing}

Respiratory specimens were obtained from children within the first 48 hours after the admission and transported to the laboratory in a viral transport medium (UTM, Copan Diagnostics, Italy). The automated nucleic acid extraction was performed according to the manufacturer's recommendations using the EZ1 Virus Mini Kit v2 (Qiagen, Luxembourg). The RT-PCR conducted on the Rotor-Gene device (Qiagen) with the FTD $\mathrm{B}$ Respiratory Pathogens 21 Kit (Luxemburg) was used to detect respiratory viruses (RSV-A/B, influenza-A/B, HRV, human coronavirus [hCoV] NL63/229E/OC43/HKU1, PIV1-4, HMPV-A/B, HBoV, human adenovirus $[\mathrm{HAdV}]$, human enterovirus $[\mathrm{EV}]$, and human parechovirus). The kits and results obtained were tested using the National External Quality Assurance Schemes (NEQUAS) External Quality Control program (NEQUAS, Scotland). 


\section{4-Statistical analysis}

Data were presented as median (interquartile ranges [IQR]) or number (\%). The categorical variables were compared between groups using the $\chi^{2}$ or Fisher Exact test. The differences in the median of the continuous variables were tested with the 2-sided Mann-Whitney or Kruskal-Wallis test. With a priori adjustment for age (categoric variables), binary logistic regression was used to predict the relationship between viral coinfection and severe LRTI. Age was entered separately into the model as a continuous and categorical variable and categorized as 1-3 months, 4-6 months, 7-12 months, 13-24 months, and 25-60 months (reference level). In the table, potential risks were presented by using categorized age. As a binary dependent variable, we assessed the severity of the disease according to the site of the hospital stay: the ICU (severe LRTI) or the short-term unit (mild LRTI). The predictors associated with the dependent variable on univariate analyses $(P<.05)$ were included in the model. As independent variables, the weight-for-height SDS, history of atopy, exposure to tobacco smoke, underlying conditions (categorized as; healthy [reference level], CLD, CHD, NMD, IS, and previous history of LRTI), neutrophil (categorized as; normal range [reference level], neutropenia, and neutrophilia) and lymphocyte (categorized as; normal range [reference level], lymphopenia, and lymphocytosis) status, and CRP value were included. Also, dummy variables to represent each level of qualitative independent variables included in the regression equation. First, virus infection type in the model was categorized as; i) single virus infection (reference level); ii) viral coinfection; iii) no-virus infection. Secondly, subgroup analyses by each of the viruses were performed to detect whether a specific virus coinfection led to severe LRTI than this specific-virus infection alone. The 13 models of comparing single and coinfection for each of the viruses were categorized as; i) specific-virus single infection (reference level); ii) specific virus and any virus coinfection; iii) single infections not including this specific virus; iv) coinfections not including this specific virus; v) no-virus infection. Finally, we conducted subgroup analyses by age for viral coinfections. The Hosmer-Lemeshow test was used to assess the goodness of fit for the model. Results were expressed as odds ratios (ORs) with $95 \%$ confidence intervals (CIs), and $P<.05$ was considered statistically significant. Statistical analyses were performed using the statistical program package SPSS (Version 22.0; IBM Corp., Armonk, NY).

\section{RESULTS}

\section{1-Study population}

We evaluated 7278 respiratory specimens during the study period. Afterward, we reviewed the patients' medical records and included $2115(29.2 \%)$ children in the study. Of these, mild and severe LRTI were observed in $562(26.6 \%)$ and $1553(73.4 \%)$ children, respectively. Seven hundred ninety-five (10.9\%) patients with a diagnosis other than LRTI, $2200(30.2 \%)$ not fulfilled criteria of LRTI according to WHO guideline, $834(11.4 \%)$ with hospital-acquired viral LRTI, $212(2.9 \%)$ with a primary bacterial infection, and 1074 $(14.8 \%)$ with a secondary bacterial infection were excluded from the study (Figure 1). Overall, the most common detected viruses were RSV (906; 42.8\%) and HRV (733; 34.7\%), followed by HBoV (212; $10.0 \%)$. We identified that HRV $(226 ; 40.2 \%$ vs. $507 ; 32.6 \% ; P=.001)$, influenza A $(73 ; 13.0 \%$ vs. $136 ; 8.8 \% ; P=$ $.004)$, and PIV3 (47; 8.4\% vs. 84; 5.4\%; $P=.01$; Supplemental Table S1) were observed more frequently in severe disease compared with mild disease.

\section{2-Viral co-infection}

Viral co-infection was present in $28.3 \%$ (599) of all children, and those with viral co-infection were at a higher risk of severe disease than those with a single virus infection (43.8\% vs. $22.7 \%$; aOR, 3.44; $95 \%$ CI, 2.74-4.53; $P<.001$; Table 1). In subgroup analyses for each virus comparing single virus infection and its co-infection; RSV (aOR, 3.70; 95\% CI, 2.62-5.23; $P<.001$ ), HRV (aOR, 3.50; 95\% CI, 2.45-5.02; $P<.001$ ), influenza A (aOR, 4.83; 95\% CI, 2.37-9.84; $P<.001$ ), PIV3 (aOR, 9.03; 95\% CI, 3.73-21.90; $P<.001$ ), HBoV (aOR, 2.37; 95\% CI, 1.27-4.63; $P=.01$ ), HAdV (aOR, 3.41; 95\% CI, 1.27-9.12; $P=.01$ ), and EV $($ aOR, 5.06; 95\% CI $1.35-18.89 ; P=.01$; Figure 2$)$ co-infections were associated with an increased risk for severe disease.

\section{3-Subgroup analyses by age}


Respiratory syncytial virus and HRV (except for between 25-60 months) coinfections with any virus were independently associated with severe disease in all age groups (Figure 3-5, Supplemental Figure S1, S2). While children between 4-24 months had a higher risk of severe infection related to PIV3 coinfection (Figure 3-5, Supplemental Figure S1), those between 7-12 months and 25-60 months had severe illness associated with influenza A coinfection (Figure 5, Supplemental Figure S2). Besides, HBoV coinfection was independently a risk factor for severe disease in children between 7-12 months (Figure 5). Unfortunately, we did not analyze the subgroup analysis for EV coinfection because of its small sample size.

\section{3-Potential risk factors}

We showed that old age (aOR, 0.98; 95\% CI, 0.97-0.99) reduced severe disease risk after the confounders adjusted. Children with higher weight-for-height SDS were significantly associated with mild disease (aOR, 0.89; 95\% CI, 0.84-0.96). Moreover, prematurity, history of atopy, exposure to tobacco smoke, underlying condition, neutrophilia, lymphopenia, and high CRP value were independent risks for severe disease (Table 1).

\section{Discussion}

This study reported that children between 1-60 months with viral coinfection increased the risk of admission to the ICU for HFNCO/BiPAP/IMV assistance. While RSV and HRV (except for between 25-60 months) coinfections were associated with severe disease in all ages, PIV3 (7-24 months) and HBoV (7-12 months) coinfections led to severe LRTI in early childhood. Besides, our study showed that influenza A coinfection was independently a risk factor for severe LRTI in children between 7-12 months and 25-60 months.

In our study, children with viral coinfection constituted $28.3 \%$ of the study population, and HBoV was the most common virus after RSV and HRV. Previous studies conducted in Barcelona and Rome ${ }^{20,21}$, where the Mediterranean climate is dominant, like Izmir, had similar results to that presented here. On the contrary, other studies reported from Canada and $\operatorname{Japan}^{7,22}$, where the continental and subtropical climate is dominant, found that viral coinfection was $17.2 \%$ and $15.8 \%$ of the patients, with the third common virus as influenza A and HMPV, respectively. These results emphasize that the seasonal distribution and frequency of viruses may vary in different countries according to their geographical and meteorological characteristics. Furthermore, the annual circulation of infections and the composition of the study population and method used in virus detection may also affect the viral coinfection rate. For example, the studies conducted in infants or hospitalized patients may determine a higher viral coinfection rate than those held in adults or population-based studies because of the more frequent detection of multiple-viruses in these patients ${ }^{21}$. The authors should interpret the viral coinfection rate carefully among different studies. Children with viral coinfection were 3.4 times more at risk of admission to the ICU than those with a single virus infection, concordant with the previous studies ${ }^{6,23}$. Several possible explanations for these results are that the direct interactions among viral genes or gene products and host immune system, the indirect interactions of host environmental changes, and the immunological interactions may determine the course of LRTI in children with viral co-infection ${ }^{24,25}$. The first virus may boost viral superinfection by consuming host defense, similar to bacterial infection ${ }^{26}$. Moreover, the coinfection of two distinct virus types may have affected the natural course of LRTI in children.

Although HRV generally causes a common cold in children ${ }^{27}$, it reduces cell proliferation and bronchial epithelial cells' self-repair capacity ${ }^{28}$. Thus, any secondary virus infection may increase the risk of severe illness. Besides, HRVs are a large group of genetically diverse RNA viruses, and HRV sub-types, particularly HRV-A and -C, have been shown to cause severe LRTI in children ${ }^{29}$. Unfortunately, we could not determine HRV sub-types in the respiratory specimens because of our virus detection method. These sub-types might have infected some severe cases. This issue stands as an interesting subject for further research. Richard et al. ${ }^{6}$ found that infants with RSV coinfection were at 2.7 times higher risk of admission to the ICU, while Semple et al. ${ }^{23}$ determined a 10 -fold higher risk of IMV in children under two years of age who had RSV and HMPV coinfection. Additionally, our results suggested that RSV coinfection remains an essential agent for severe LRTI not only in children younger than 24 months but also in those beyond 24 months. Since 
there is no existing vaccine for RSV, the consideration of passive immunoprophylaxis during RSV season may protect children with an underlying medical condition from severe RSV co-infections ${ }^{17}$.

Coinfection with other viruses is very common during influenza infection ${ }^{30}$. In recent meta-analyses ${ }^{31,32}$, COVID-19 coinfection was identified more commonly with influenza A and RSV. Although it was suggested that children with COVID-19 had a better prognosis than adults, influenza A coinfection with COVID-19 may inhibit the host's immune system, increase antibacterial therapy intolerance, and be harmful disease's prognosis $^{31}$. Besides, secondary bacterial infections may exist at a rate of $40 \%$ in influenza A infections ${ }^{33}$. Therefore, we note that any secondary bacterial infection, which was not detected, might increase influenza A's severity. However, a comprehensive and extensive sample size study should be needed. Although there is no licensed influenza vaccine for children under six months, alternative strategies, including maternal vaccination during pregnancy and household vaccination, might reduce severe influenza infections. Increasing influenza and bacterial vaccination, with environmental precautions such as frequent hand washing, decontamination of hands, and cleaning concrete surfaces with water and disinfectants, are essential to prevent transmission of the respiratory viruses and reduce the severe disease burden. The efficacy of neuraminidase inhibitors in healthy children is limited and does not recommend general treatment ${ }^{34}$. Nevertheless, early initiation of neuraminidase inhibitors is associated with shorter symptoms, decreased complications, and hospitalization ${ }^{34}$.

Human bocavirus was first identified in children's respiratory tract in $2005 ; 75 \%$ of the HBoV infections are associated with multiple viral infections ${ }^{16}$. $\mathrm{HBoV}$ infection usually results in a mild, self-limiting respiratory tract infection and might even be asymptomatic ${ }^{4}$. However, several case reports reported that HBoV coinfection with other viruses could cause complications such as pneumothorax, pneumomediastinum, and severe respiratory failure requiring ICU/IMV ${ }^{35}$. Slow elimination of the viral antigens by the immature immune system might explain the coinfection with $\mathrm{HBoV}$ and the severity of the illness in infants. Considering the National Respiratory and Enteric Viruses Surveillance System study conducted from 1990 to $2004^{36}$, PIV3 (52\%) was the most frequently determined serotype. In the US, the estimated LRTI and hospitalization related to PIV3 were reported at 1.1million and 29.000, respectively ${ }^{37}$. Parainfluenza virus 3 leads to LRTI more common than other serotypes in neonates and infants and is clinically indistinguishable from RSV infection. Additionally, PIV and RSV belong to the Paramyxoviridae family, enveloped RNA, similar epidemiologic, and clinical outcomes ${ }^{22}$. Thus, children aged younger than 24 months with PIV3 coinfection may need equal medical attention to those with RSV coinfection regarding disease severity.

Our regression analysis determined that young age, prematurity, malnutrition, exposure to tobacco smoke, and atopy history were independent risk factors for severe LRTI, aligned with earlier studies ${ }^{6,15}$. Young age and prematurity cause more severe respiratory stress because of the limited aerobic respiratory capacity associated with the relatively smaller airway size, the immune system's naivety, and less strength and respiratory muscle endurance ${ }^{24}$. Malnutrition may result in secondary immunosuppression, atrophy of the respiratory muscles, and inadequate muscle contraction because of the electrolyte disturbance ${ }^{15}$. Taking measures to prevent malnutrition, such as providing information and supporting the mother about breastfeeding, using prophylactic vitamin D and iron supplements, and a regular follow-up of the anthropometric measurements, may protect children from severe disease.

An unanswered question has been whether the presence of a pre-existing abnormality of the immune response in some infants leads to severe illness. A recently published study has demonstrated that both viral infection and allergic sensitization are strongly correlated with asthma development after six years old in children ${ }^{14}$. Furthermore, severe disease is found to be related to allergic sensitization before symptomatic HRV infection. Our results showing that allergic sensitivity increases the disease's severity is one of our relevant findings, which are rarely supported by the literature but can shed light on studies investigating this relationship. Tobacco smoking continues to be a fundamental health problem worldwide, and a recently published metaanalysis has confirmed the effect of exposure to tobacco smoke on developing LRTI in children ${ }^{38}$. Increasing comprehensive bans on tobacco advertising, promotion, sponsorship, and tobacco taxes might reduce tobacco consumption. Additionally, ample pictorial or graphic health warnings, with hard-hitting messages, might 
convince smokers to protect the health of non-smokers by not smoking inside the home ${ }^{38}$.

In children with CLD, the increased inflammatory markers, impaired airway anatomy, and mucociliary clearance could support the progression to severe LRTI, whereas altered pulmonary mechanics, cyanosis, pulmonary hypertension, and ventilation-perfusion mismatch in children with CHD could increase the severity of the disease. Children with NMD expose severe illness because of impaired or inadequate mucociliary activity and immobility. These findings are essential when considering the increased survival of children with a chronic underlying condition. The presence of neutrophilia, lymphopenia, and high CRP values were independent risks for severe disease. Previous studies have shown that many neutrophils detected during influenza A and RSV infections are associated with a more severe condition, and lymphopenia increases virus replication $^{39,40}$. The prognosis can be predicted to be more severe in children with neutrophilia, lymphopenia, and high CRP values. However, larger-population studies are required to monitor children with LRTI according to hematologic values.

Our study's limitations include those related to retrospective studies, including bias regarding patient selection and accuracy related to the medical record. To minimize bias, we developed a standardized data form to guide data collection, only included patients with the medical records' information, and the same experienced clinician performed data collection. Second, patients who visit the hospital later may present with more severe signs and symptoms than those who visit the hospital immediately after illness onset. However, we could not compare this potential effect on disease severity because of the study's retrospective design. Third, including only children presented to the tertiary hospital might have resulted in the study population's heterogeneity. Finally, a reliable test for bacterial co-detection was not available at the time of the study, which could cause that we might have underestimated the impact of bacteria on severe LRTI.

In conclusion, children between 1-60 months hospitalized with LRTI and detected viral coinfection were at about 3.5 times higher risk for $\mathrm{HFNCO/BiPAP/IMV} \mathrm{assistance.} \mathrm{Respiratory} \mathrm{syncytial} \mathrm{virus} \mathrm{and} \mathrm{HRV}$ (except for between 25-60 months) coinfections caused severe LRTI in all age groups, whereas PIV3 (424 months) and HBoV (7-12 months) coinfections were associated with severe LRTI in early childhood. Additionally, influenza A coinfection led to severe LRTI in children between 7-12 months and 25-60 months.

\section{Acknowledgments}

Author contributions:

Dr. Eşki: Designed the study and contributed to the writing of the manuscript, patient recruitment; data collection, analysis, and interpretation; and manuscript review.

Dr. Kartal Öztürk: Contributed to patient recruitment, data analysis and interpretation, and manuscript review.

Dr. Çiçek: Contributed to data collection, analysis, and interpretation, and manuscript review.

Dr. Gülen: Contributed to data collection, analysis, and interpretation, and manuscript review.

Dr. Demir: Contributed to the writing of the manuscript; patient recruitment; data collection, analysis, and interpretation; and manuscript review.

Financial/nonfinancial disclosures: The authors have reported to Pediatric Pulmonology Journal that no potential conflicts of interest exist with any companies/organizations whose products or services may be discussed in this article.

Role of sponsors: This study has no sponsors.

Figure 1. The study flow diagram

Figure 2. Comparison of virus co-infection with single virus infection

Figure 3. Subgroup regression analysis of each virus co-infection by age between 1-3 months 
Figure 4. Subgroup regression analysis of each virus co-infection by age between 4-6 months

Figure 5. Subgroup regression analysis of each virus co-infection by age between 7-12 months

Supplemental Figure S1. Subgroup regression analysis of each virus co-infection by age between 13-24 months

Supplemental Figure S2. Subgroup regression analysis of each virus co-infection by age between 25-60 months

\section{References}

1. Ruuskanen O, Lahti E, Jennings LC, Murdoch DR. Viral pneumonia. Lancet 2011;377(9773):1264-1275.

2. Singleton RJ, Holman RC, Folkema AM, Wenger JD, Steiner CA, Redd JT. Trends in lower respiratory tract infection hospitalizations among American Indian/Alaska Native children and the general US child population. J Pediatr 2012;161(2).

3. Hasegawa K, Tsugawa Y, Brown DFM, Mansbach JM, Camargo CA. Trends in bronchiolitis hospitalizations in the United States, 2000-2009. Pediatrics 2013;132(1):28-36.

4. Florin TA, Plint AC, Zorc JJ. Viral bronchiolitis. Lancet 2017;389(10065):211-224.

5. Asner SA, Science ME, Tran D, Smieja M, Merglen A, Mertz D. Clinical disease severity of respiratory viral coinfection versus single viral infection: A systematic review and meta-analysis. PLoS One 2014;9(6).

6. Richard N, Komurian-Pradel F, Javouhey E, Perret M, Rajoharison A, Bagnaud A, Billaud G, Vernet G, Lina B, Floret D, et al. The impact of dual viral infection in infants admitted to a pediatric intensive care unit associated with severe bronchiolitis. Pediatr Infect Dis J 2008;27(3):213-217.

7. Brand HK, De Groot R, Galama JMD, Brouwer ML, Teuwen K, Hermans PWM, Melchers WJG, Warris A. Infection with multiple viruses is not associated with increased disease severity in children with bronchiolitis. Pediatr Pulmonol 2012;47(4):393-400.

8. Eşki A, Öztürk GK, Gülen F, Çiçek C, Demir E. Risk factors for infuenza virus related severe lower respiratory tract infection in children. Pediatr Infect Dis J 2019;38(11):1090-1095.

9. Scotta MC, Chakr VCBG, de Moura A, Becker RG, de Souza APD, Jones MH, Pinto LA, Sarria EE, Pitrez PM, Stein RT, et al. Respiratory viral coinfection and disease severity in children: A systematic review and meta-analysis. J Clin Virol 2016;80:45-56.

10. Goka EA, Vallely PJ, Mutton KJ, Klapper PE. Single and multiple respiratory virus infections and severity of respiratory disease: A systematic review. Paediatr Respir Rev 2014;15(4):363-370.

11. WHO. Pocket Book of Hospital Care for Children: Guidelines for the Management of Common Childhood Illnesses. 2013.

12. Raymond J, Aujard Y. Nosocomial Infections in Pediatric Patients A European, Multicenter Prospective Study. Infect Control 2000;21(4):260-263.

13. Asner SA, Rose W, Petrich A, Richardson S, Tran DJ. Is virus coinfection a predictor of severity in children with viral respiratory infections? Clin Microbiol Infect 2015;21(3):264.e1-264.e6.

14. Jackson S, Mathews KH, Pulanić D, Falconer R, Rudan I, Campbell H, Nair H. Risk factors for severe acute lower respiratory infections in children - a systematic review and meta-analysis. Croat Med J 2013;54(2):110-121.

15. Sonego M, Pellegrin MC, Becker G, Lazzerini M. Risk factors for mortality from acute lower respiratory infections (ALRI) in children under five years of age in low and middle-income countries: A systematic review and meta-analysis of observational studies. PLoS One 2015;10(1). 
16. Mazur NI, Bont L, Cohen AL, Cohen C, Von Gottberg A, Groome MJ, Hellferscee O, Klipstein-Grobusch K, Mekgoe O, Naby F, et al. severity of respiratory syncytial virus lower respiratory tract infection with viral coinfection in HIV-uninfected children. Clin Infect Dis 2017;64(4):443-450.

17. Statement P. Updated Guidance for Palivizumab Prophylaxis Among Infants and Young Children at Increased Risk of Hospitalization for Respiratory Syncytial Virus Infection. 2017.

18. Das MK, Bhattacharyya N, Bhattacharyya AK. WHO child growth standards. 2010.

19. Lanzkowsky's Manual of Pediatric Hematology and Oncology. 2016.

20. Cangiano G, Nenna R, Frassanito A, Evangelisti M, Nicolai A, Scagnolari C, Pierangeli A, Antonelli G, Papoff P, Petrarca L, et al. Bronchiolitis: Analysis of 10 consecutive epidemic seasons. Pediatr Pulmonol 2016;51(12):1330-1335.

21. Martínez-Roig A, Salvadó M, Caballero-Rabasco MA, Sánchez-Buenavida A, López-Segura N, BonetAlcaina M. Viral Coinfection in Childhood Respiratory Tract Infections. Arch Bronconeumol 2015;51(1):5-9.

22. Harada Y, Kinoshita F, Yoshida LM, Le Nhat M, Suzuki M, Morimoto K, Toku Y, Tomimasu K, Moriuchi $\mathrm{H}$, Ariyoshi K. Does respiratory virus coinfection increases the clinical severity of acute respiratory infection among children infected with respiratory syncytial virus? Pediatr Infect Dis J 2013;32(5):441-445.

23. Semple MG, Cowell A, Dove W, Greensill J, McNamara PS, Halfhide C, Shears P, Smyth RL, Hart CA. Dual Infection of Infants by Human Metapneumovirus and Human Respiratory Syncytial Virus Is Strongly Associated with Severe Bronchiolitis. J Infect Dis 2005;191(3):382-386.

24. Hammer J, Numa A, Newth CJL. Acute respiratory distress syndrome caused by respiratory syncytial virus. Pediatr Pulmonol 1997;23(3):176-183.

25. DaPalma T, Doonan BP, Trager NM, Kasman LM. A systematic approach to virus-virus interactions. Virus Res 2010;149(1):1-9.

26. McCullers JA. The co-pathogenesis of influenza viruses with bacteria in the lung. Nat Rev Microbiol 2014;12(4):252-262.

27. Costa LF, Oliveira Queiróz DA, Lopes Da Silveira H, Bernardino Neto M, De Paula NT, Oliveira TFMS, Tolardo AL, Yokosawa J. Human rhinovirus and disease severity in children. Pediatrics 2014;133(2).

28. Xatzipsalti M, Psarros F, Konstantinou G, Gaga M, Gourgiotis D, Saxoni-Papageorgiou P, Papadopoulos NG. Modulation of the epithelial inflammatory response to rhinovirus in an atopic environment. Clin Exp Allergy 2008;38(3):466-472.

29. Lee WM, Lemanske RF, Evans MD, Vang F, Pappas T, Gangnon R, Jackson DJ, Gern JE. Human rhinovirus species and season of infection determine illness severity. Am J Respir Crit Care Med 2012;186(9):886891.

30. de Souza WM, Buss LF, Candido D da S, Carrera JP, Li S, Zarebski AE, Pereira RHM, Prete CA, de Souza-Santos AA, Parag K V., et al. Epidemiological and clinical characteristics of the COVID-19 epidemic in Brazil. Nat Hum Behav 2020;4(8):856-865.

31. Zimmermann P, Curtis N. COVID-19 in Children, Pregnancy and Neonates: A Review of Epidemiologic and Clinical Features. Pediatr Infect Dis J 2020:469-477.

32. Lansbury L, Lim B, Baskaran V, Lim WS. Coinfections in people with COVID-19: a systematic review and meta-analysis. J Infect 2020;81(2):266-275.

33. Shah NS, Greenberg JA, McNulty MC, Gregg KS, Riddell J, Mangino JE, Weber DM, Hebert CL, Marzec NS, Barron MA, et al. Bacterial and viral coinfections complicating severe influenza: Incidence and impact among 507 U.S. patients, 2013-14. J Clin Virol 2016;80:12-19. 
34. Jefferson T, Jones MA, Doshi P, Del Mar CB, Hama R, Thompson MJ, Spencer EA, Onakpoya IJ, Mahtani KR, Nunan D, et al. Neuraminidase inhibitors for preventing and treating influenza in adults and children. Cochrane Database Syst Rev 2014;2014(4).

35. Uršič T, Steyer A, Kopriva S, Kalan G, Krivec U, Petrovec M. Human bocavirus as the cause of a life-threatening infection. J Clin Microbiol 2011;49(3):1179-1181.

36. Knott AM, Long CE, Hall CB. Parainfluenza viral infections in pediatric outpatients: Seasonal patterns and clinical characteristics. Pediatr Infect Dis J 1994;13(4):269-273.

37. Lee MS, Walker RE, Mendelman PM. Medical burden of respiratory syncytial virus and parainfluenza virus type 3 infection among US children. Implications for design of vaccine trials. Hum Vaccin 2005;1(1):611.

38. WHO. WHO report on the global tobacco epidemic. 2008 [accessed 2020 May 27]. https://www.who.int/tobacco/surveillance/policy/country_profile/tur.pdf?ua=1

39. Browne MJ. Comparative inhibition of influenza and parainfluenza virus replication by ribavirin in MDCK cells. Antimicrob Agents Chemother 1981;19(5):712-715.

40. O'Donnell DR, Carrington D. Peripheral blood lymphopenia and neutrophilia in children with severe respiratory syncytial virus disease. Pediatr Pulmonol 2002;34(2):128-130.

\section{Hosted file}

Table 1.pdf available at https://authorea.com/users/390866/articles/505089-is-viral-coinfection-a-risk-factor-for-severe-lower-respiratory-tract-infection-a-retrospectiveobservational-study 


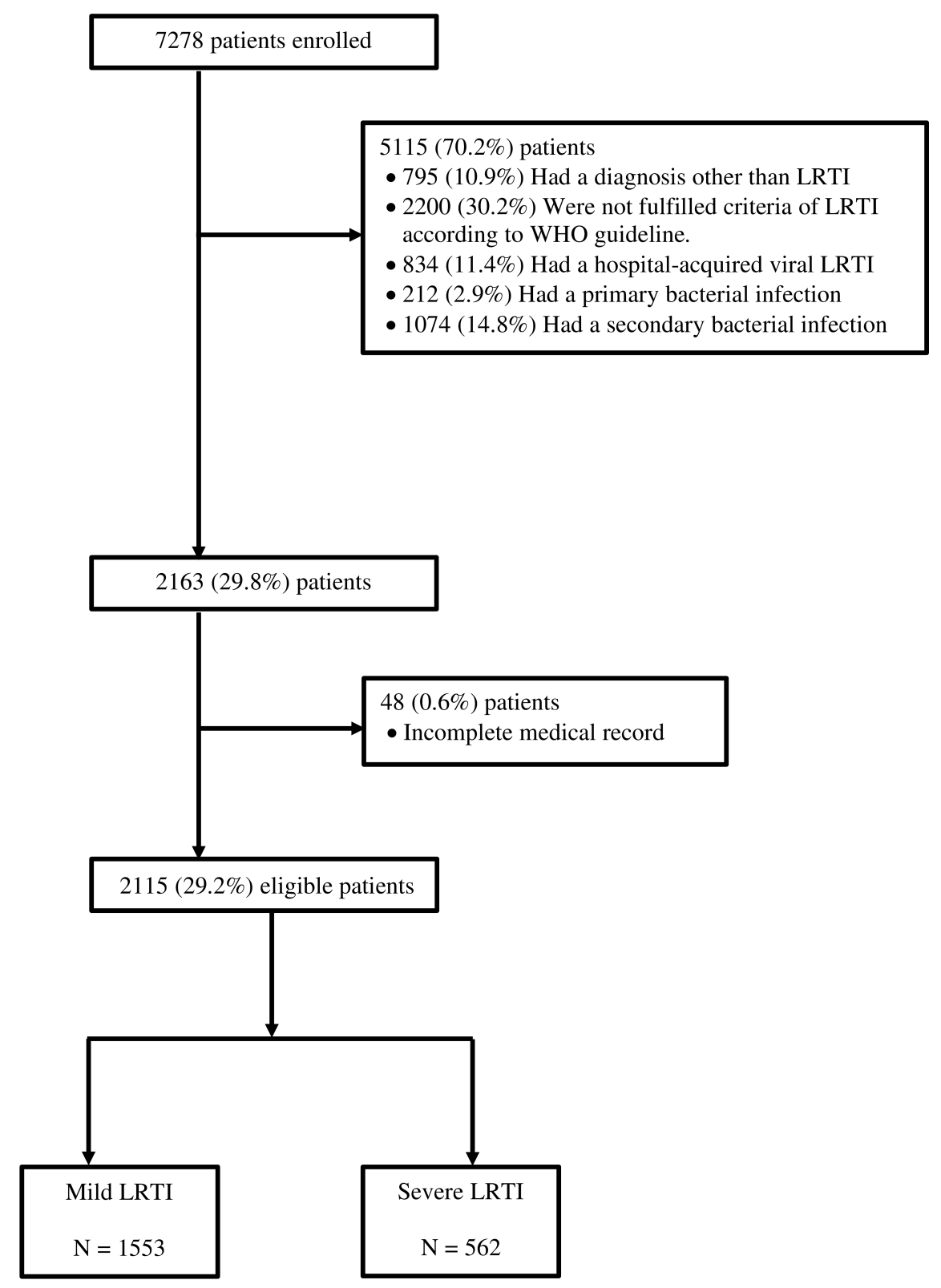

LRTI, lower respiratory tract infection; WHO, world health organization. 


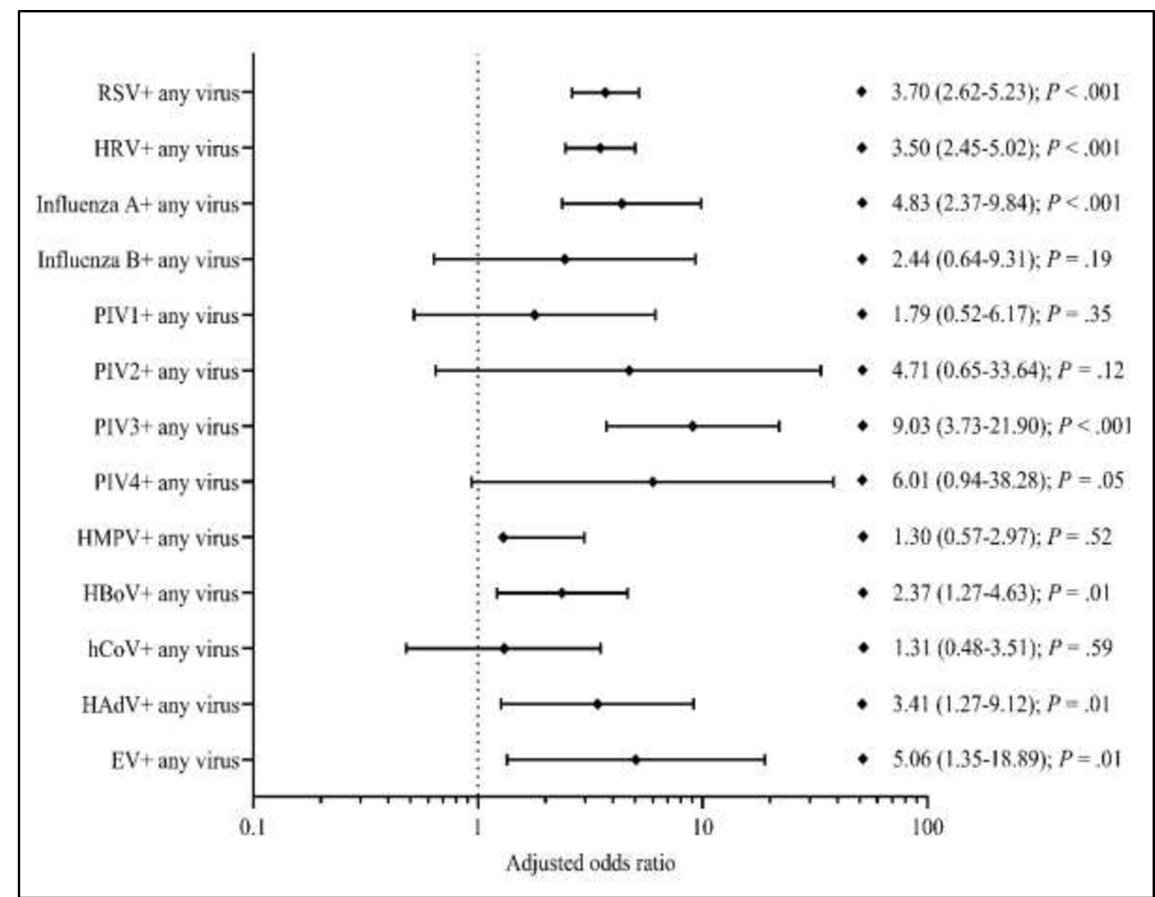

$N=2115$ patients, within 38 missing values; 2077 patients were included in all multivariate analyses.

Number of each virus as a single and coinfection; RSV (604 vs. 292; adjusted $R^{2}=.208$ ), HRV (84 vs. 323 ; adjusted $R^{2}=.206$ ), influenza A (84 vs. 112 ; adjusted $R^{2}=.205$ ), influenza B (31 vs. 26; adjusted $R^{2}=.207$ ), PIV1 (25 vs. 25; adjusted $R^{2}=.207$ ), PIV2 (12 vs. 13; adjusted $R^{2}$ $=.205$ ), PIV3 (66 vs. 63 ; adjusted $R^{2}=.209$ ), HMPV (78 vs. 60; adjusted $R^{2}=.209$ ), HBoV (66 vs. 138; adjusted $R^{2}=.209$ ), hCoV (27 vs. 77; adjusted $\left.R^{2}=.208\right)$, HAdV (32 vs. 105; adjusted $R^{2}=.206$ ), and EV (19 vs. 30 ; adjusted $R^{2}=.207$ ).

RSV, respiratory syncytial virus; HRV, human rhinovirus; HBoV, human bocavirus; HMPV, human metapneumovirus; HAdV, human adenovirus; PIV1-4, parainfluenza virus 1-4; hCoV, human coronavirus; EV, human enterovirus. 


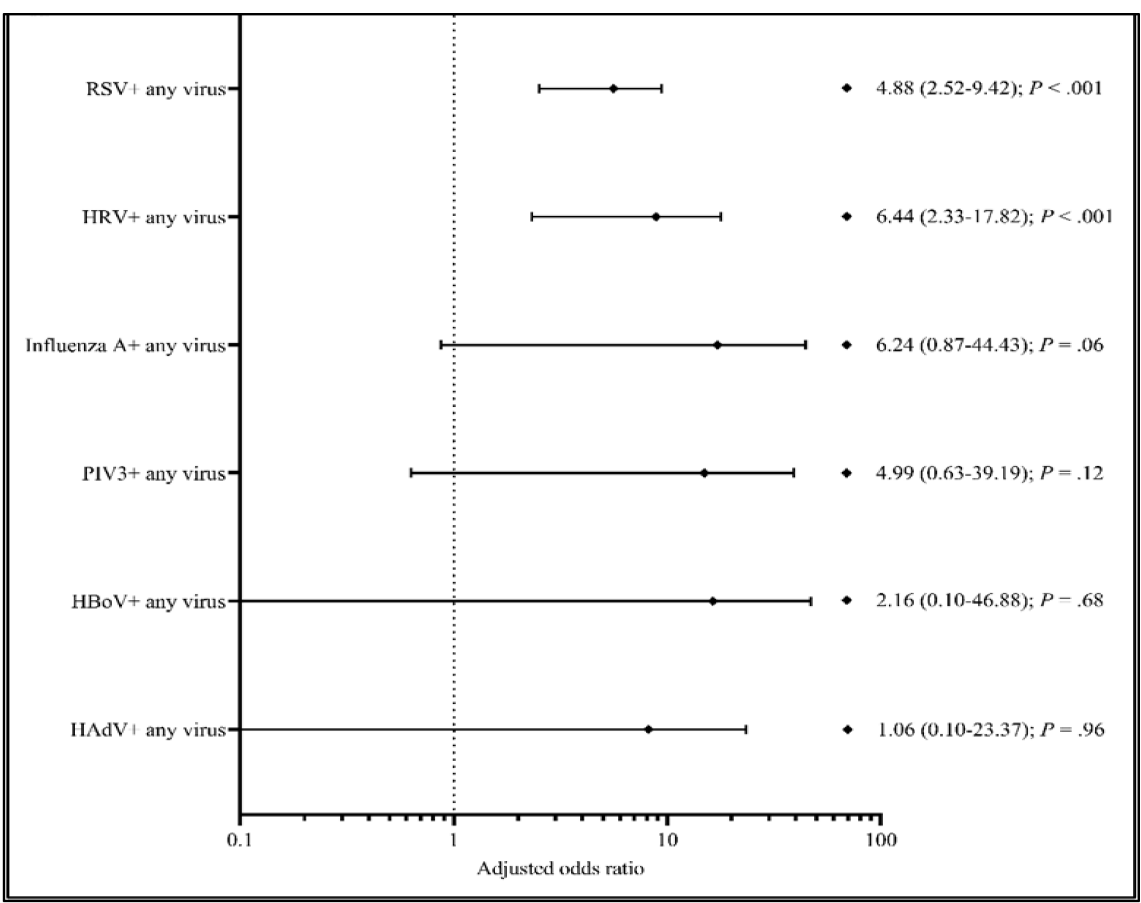

$N=528$ patients, within two missing values; 526 patients were included in the multivariate analysis $\left(R^{2}\right.$ for each viruses; $\mathrm{RSV}=.413 ; \mathrm{HRV}=.414$; influenza $\mathrm{A}$ and $\mathrm{HBoV}=.410 ; \mathrm{PIV} 3$ and HAdV $=.411)$.

Because of the small sample sizes, human enterovirus was not calculated.

RSV, respiratory syncytial virus; HRV, human rhinovirus; PIV3, parainfluenza virus 3 ; HBoV, human bocavirus; HAdV, human adenovirus. 


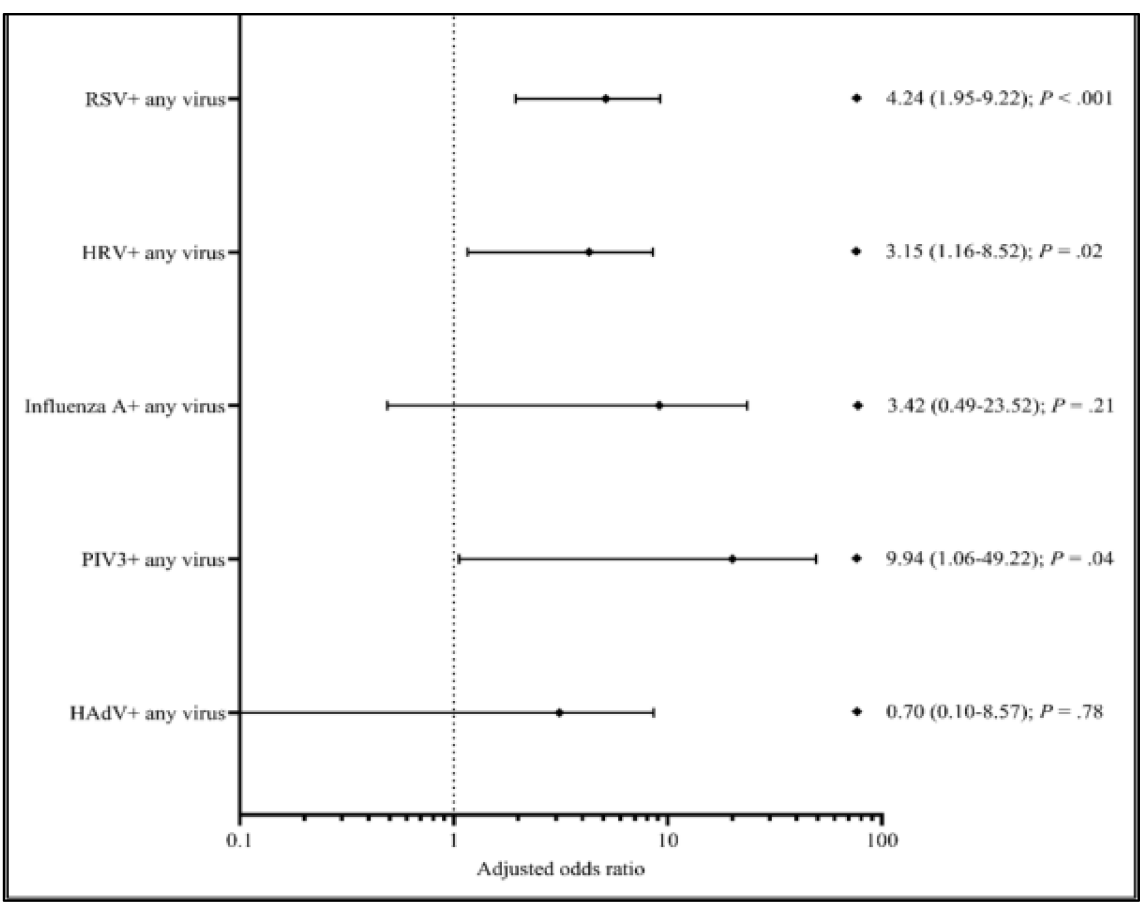

$N=347$ patients, within 8 missing values; 339 patients were included in the multivariate analysis $\left(R^{2}\right.$ for each viruses; RSV and PIV3 $=.283$; HRV and influenza $\mathrm{A}=.279 ; \mathrm{HAdV}=$ $.288)$.

Because of the small sample sizes, human enterovirus and bocavirus were not calculated.

RSV, respiratory syncytial virus; HRV, human rhinovirus; PIV3, parainfluenza virus; HAdV, human adenovirus. 


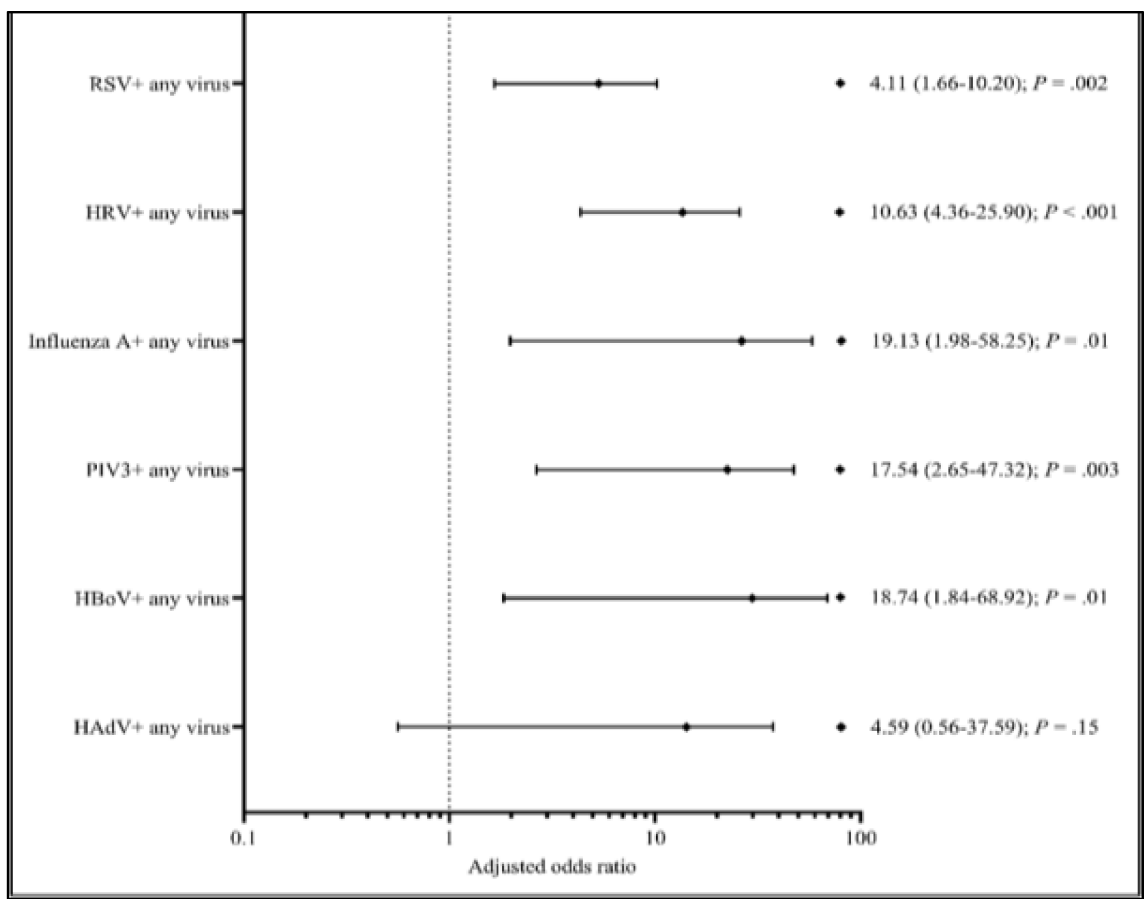

$N=426$ patients, within ten missing values; 418 patients were included in the multivariate analysis $\left(R^{2}\right.$ for each viruses; $\mathrm{RSV}=.293 ; \mathrm{HRV}=.295$; influenza $\mathrm{A}=.289 ; \mathrm{PIV} 3=.292 ; \mathrm{HBoV}$ $=.297 ; \mathrm{HAdV}=.290)$.

Because of the small sample sizes, human enterovirus was not calculated.

RSV, respiratory syncytial virus; HRV, human rhinovirus; PIV3, parainfluenza virus 3 ; HBoV, human bocavirus; HAdV, human adenovirus. 